\title{
Channeling process in a bent crystal
}

\author{
Andrey V. Solov'yov, ${ }^{*}$ Andreas Schäfer, and Walter Greiner \\ Institut für Theoretische Physik der Universität, 6000 Frankfurt am Main, Germany
}

(Received 14 March 1995)

\begin{abstract}
We have investigated the channeling process of charged particles in a bent crystal. Invoking simple assumptions we derive a criterion, which determines whether channeling occurs or not. We obtain the same criterion using the Dirac equation. It is shown that the centrifugal force acting on the particle in the bent crystal significantly alters the effective transverse potential. The cases of axial and planar channeling are considered. The channeling probability and the dechanneling probability due to tunneling of the particle under the barrier in the effective transverse potential are estimated. These probabilities depend on the specific scaling parameter characterizing the process. Using the quasiclassical theory of synchrotron radiation we have calculated the contribution to the radiation spectrum, which arises due to the curvature of the channel. This contribution becomes significant for $\mathrm{TeV}$ electrons or positrons. Some practical consequences of our results are briefly discussed.
\end{abstract}

PACS number(s): 41.75.-i, 61.85.+p, 03.65.-w

\section{INTRODUCTION}

Channeling was discovered in the early 1960 s by computer simulations of ion motion in crystals $[1,2]$. Large penetration lengths were obtained for ions incident along crystallographic directions of low indexes. Such a guided motion had already been predicted [3] by Stark in 1912 . A comprehensive theoretical study [4,5] introduced the important continuum approximation for the interaction potentials between energetically charged particles and lattice atoms arranged in atomic strings and planes, respectively. These concepts were subsequently widely used in the interpretation of channeling experiments (see, e.g., the review article [6]). The idea of channeling was explored in different physical processes. As argued in [7] one could collide two electron beams under channeling condition. The possibility that high-energy photons are channeled when passing through an oriented single crystal was investigated in Ref. [8].

Radiation phenomena during the interaction of positrons and electrons with crystals had already been investigated before channeling was discovered. Frisch and Olsen [9] observed an enhancement in the soft quanta region for $1 \mathrm{GeV}$ electrons aligned with the $\langle 110\rangle$ axis in a germanium crystal. Numerous theoretical and experimental investigations in this field were subsequently performed (for review see also, e.g., [10]).

Experiments with electrons in the $\mathrm{GeV}$ range [11] as well as in the $\mathrm{MeV}$ range [12] have shown that the observed radiation spectra are in agreement with the general predictions given in $[13,14]$ concerning the energy dependence of intensity and photon energies. Because of

\footnotetext{
*Permanent address: A.F. Ioffe Physical-Technical Institute of the Academy of Sciences of Russia, 194021 St. Petersburg, Russia.
}

the intensive theoretical and experimental investigation further review articles were published $[15,16]$.

One of the most powerful and successful methods in the theory of high-energy channeling is the semiclassical method. The semiclassical method was developed in Ref. [17]. Using this method the spectra of photons and electron-positron pairs were successfully described. The quasiclassical method was also successfully applied in a number of papers $[18,19]$ to describe the position of the experimentally observed peak in the spectrum of 150 $\mathrm{GeV}$ electron energy loss in a thin germanium crystal. This calculation was also done purely quantum mechanically in [20].

Channeling was also successfully used to bend ion beams [21-24]. The basic effect is that charged particles traveling through a crystal nearly parallel to a crystal axis experience the collective electric field of the ions and are thus steered into the interatomic region (for positively charged particles) or into the vicinity of the atoms (for negatively charged particles). Due to this effect ion beams can be bent much more strongly than with external electric or magnetic fields. The efficiency is, however, typically smaller than $10 \%$. In Ref. [25] the question of whether the channeling effect can also be used to focus beams, especially ion beams, was discussed. To this end a crystal is needed in which the crystal axes are no longer parallel, but are slanted more and more the farther away they are from the axes of the beam. Then the bending angle of particles far away from the beam axes would be largest and a general focusing effect would result. Such a crystal can in principle be produced by varying the germanium to silicon ratio in a mixed crystal [25].

In the present paper we investigate the channeling process of electrons and ions in a bent crystal. We consider a charged particle moving along the bent channel. Using the Dirac equation we calculate the probability of channeling and investigate its dependence on the characteristic parameters. We describe the following specific 
features of the channeling process in a bent crystal: the alteration of the transverse potential due to the centrifugal force acting on the particle, the polarization of the states of the transverse motion, and the tunneling of the particle through the barrier in the effective transverse potential. Within the framework of the quasiclassical method [17] we calculate the spectrum of radiation originating due to the bending of the particle's trajectory. This method treats the motion of the charged particle in the electric field quasiclassically. It takes into account the quantum corrections to the motion of the particle as well as the recoil effect during the radiation of the photon. Therefore the results of the calculation are applicable even for photon energies comparable to the energy of the incident particle. We demonstrate in our paper that such high-energy photons can be emitted in the electric field of the bent channel, if the energy of the particle is sufficiently large. For an electron or a positron the energy must be in the $\mathrm{TeV}$ range, which should be accessible in colliders of the next generation. We consider in our paper the cases of axial and planar channeling.

\section{CHANNELING CONDITION IN A BENT CRYSTAL}

\section{A. Qualitative consideration}

If a fast charged particle moves at a very small angle relative to crystal axes or a crystal plane, then the transverse motion of the particle is strongly influenced by the field of the crystal (see, e.g., $[16,17]$ ). As was demonstrated by Lindhard [4], the condition for the particle to be trapped in the transverse directions by the field of the channel is

$$
\Theta \leq \Theta_{L}=\sqrt{\frac{2 U_{0}}{m_{0} c^{2} \gamma}} .
$$

In this equation $\Theta$ is the angle between the direction of the incident beam and the crystal axes or the crystal plane, $\Theta_{L}$ is the critical Lindhard angle; $U_{0}$ is the depth of the transverse effective potential; $m_{0}$ is the mass of the particle; $c$ is the velocity of light; $\gamma$ is the relativistic Lorentz factor. This condition arises from the requirement that the transverse kinetic energy of the particle in the channel has to be less than the depth of the transverse potential.

Now let us assume that the channel has a certain curvature radius equal to $\rho$. Moving along the channel a particle oscillates in the transverse plane as shown in Fig. 1. It is qualitatively clear that the channeling effect in the bent crystal takes place if the bending angle $\Theta_{1}$ of the channel for one period of the transverse oscillation of the particle is much smaller than the Lindhard angle:

$$
\Theta_{1} \ll \Theta_{L}
$$

Using simple relations between the variables, characterizing the process

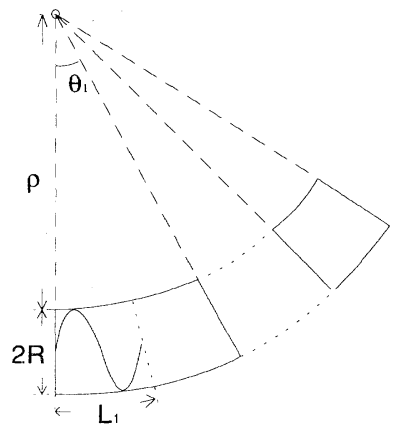

FIG. 1. Oscillations of the particle in the electric field of a bent channel.

$$
\begin{aligned}
& L_{1}=\rho \Theta_{1}, \quad L_{1}=c T_{1}, \quad T_{1}=\frac{2 R}{v_{\perp}}, \\
& v_{\perp} \sim \sqrt{2 m U_{0}}, \quad m=m_{0} \gamma
\end{aligned}
$$

we derive from (2) the condition

$$
C=\frac{\varepsilon_{||} R}{U_{0} \rho} \ll 1 .
$$

Here $\varepsilon_{\|} \approx m_{0} c^{2} \gamma$ is the kinetic energy of the longitudinal motion of the particle in the channel. We shall see that any physical quantity characterizing the channeling process in a bent crystal is a function of the parameter $C$, appearing in (4).

The total bending angle of the particle passing through a crystal of length $L$ can be estimated as follows:

$$
\Theta_{\max } \leq \Theta_{L} N_{o s c}
$$

where $N_{\text {osc }}$ is the number of transverse oscillations the particle makes on the length of the channel. It can be estimated as

$$
N_{\text {osc }} \sim \frac{L}{L_{1}}=\frac{L}{R} \sqrt{\frac{U_{0}}{2 \varepsilon_{\|}}}
$$

and the inequality $(5)$ reads

$$
\Theta_{\max } \leq \frac{U_{0}}{\varepsilon} \frac{L}{R}=\frac{1}{C} \frac{L}{\rho} .
$$

This equation shows that the large bending angle $\Theta_{\max } \sim 1$ of the channeling particles can be achieved even for the small fractions $L / \rho \ll 1$, if the main condition of channeling (4) is well fulfilled. The important feature of $(7)$ is that the maximum bending angle does not depend on the curvature $\rho$ of the channel, but is determined by its total length.

Now let us derive the same conditions considering the dynamic of a particle in the field of a bent channel. It is qualitatively clear that the centrifugal force $F_{1}$ acting on the particle must be compensated by the transverse binding force of the channel $F_{2}$ :

$$
F_{1} \leq F_{2}
$$


where

$$
\begin{gathered}
F_{1}=m a, \quad a=\frac{v^{2}}{\rho}, \quad m=m_{0} \gamma \\
F_{2}=\left|\vec{\nabla}_{\perp} U\left(r_{\perp}\right)\right| .
\end{gathered}
$$

Substituting (9) into (8) and taking into account that $\rho=L / \Theta_{\max }$, we obtain

$$
\Theta_{\max } \leq \frac{\left|\nabla_{\perp} U\left(r_{\perp}\right)\right|}{m_{0} c^{2} \gamma} L
$$

Estimating $\left|\nabla_{\perp} U\left(r_{\perp}\right)\right| \sim U_{0} / R$ we derive that the condition (10) is equivalent to (7). It is clear that it can also be expressed in the form (5).

The given conditions are applicable both for axial and planar channeling. In the latter case $\varepsilon_{\|}$must be considered as the energy of the longitudinal motion in the direction of the plane.

To get some feeling for the typical quantities characterizing the channeling process let us substitute $L \sim 2 \times 10^{-2}$ $\mathrm{cm}, R \sim 2 \AA, U_{0} \sim 100 \mathrm{eV}$, and $E \sim 100 \mathrm{GeV}$ in (1), $(4),(6)$, and (7). Then we get

$$
\Theta_{L} \sim 5 \times 10^{-5}, \quad N_{o s c} \sim 60, \quad \Theta_{\max } \leq 3 \times 10^{-3} .
$$

\section{B. The equation of motion}

To derive the conditions proposed in the preceding section let us consider the Dirac equation for a particle moving in a bent channel. Since we are not going to analyze in this paper the spin motion of the particle, it is more convenient to start our consideration with the second order Dirac equation [26]:

$$
\left\{-\hbar^{2} \Delta-p^{2}+\frac{2 e}{c^{2}} \varepsilon \Phi-\frac{e^{2}}{c^{2}} \Phi^{2}+i \frac{e \hbar}{c} \vec{\alpha} \cdot \vec{E}\right\} \Psi(\vec{r})=0 .
$$

Here $\varepsilon, p$ are the total energy and the momentum of the particle; $\varepsilon^{2} / c^{2}-p^{2}=m_{0}^{2} c^{2} ; \Phi=\Phi(\vec{r})$ is the potential of the channel; $\vec{E}=-\vec{\nabla} U(\vec{r}), U(\vec{r})=e \Phi ; \vec{\alpha}=\gamma^{0} \vec{\gamma}$. Equation (11) includes the static electric field of the channel. The magnetic field is neglected.

This form of the Dirac equation is convenient for the treatment of the spin-moment coupling, which is described by the last term in (11). This term as well as the previous one does not depend on the energy $\varepsilon$, while potential is proportional to $\varepsilon$. Keeping only the last term for $\varepsilon \gg|U(r)|$ leads to the nonrelativistic Schrödinger equation

$$
\left\{-\frac{\hbar^{2}}{2 m} \Delta+U(\vec{r})\right\} \Psi(\vec{r})=\frac{p^{2}}{2 m} \Psi(\vec{r}) .
$$

Let us note that the momentum $p$ and the mass of the particle are relativistic: $p^{2}=m_{0}^{2}+\varepsilon^{2} / c^{2}$ and $m=m_{0} \gamma$.

The separation of the transverse and the longitudinal motion in this equation is trivial in the case of linear channeling. In this case the potential depends only on the transverse coordinate $\vec{r}_{\perp}$ and therefore the solution of (12) is of the form

$$
\begin{array}{r}
\Psi(\vec{r})=\exp \left\{i \vec{p}_{\|} \vec{r}\right\} \varphi\left(\vec{r}_{\perp}\right), \\
\varepsilon=\varepsilon_{\perp}+\varepsilon_{\|},
\end{array}
$$

where $\varphi\left(\vec{r}_{\perp}\right)$ is the solution of the Schrödinger equation for the particle with the relativistic mass $m=m_{0} \gamma$ :

$$
\left\{-\frac{\hbar^{2}}{2 m} \Delta_{\vec{r}_{\perp}}+U\left(\vec{r}_{\perp}\right)\right\} \Psi\left(\vec{r}_{\perp}\right)=\varepsilon_{\perp} \Psi\left(\vec{r}_{\perp}\right) .
$$

Now let us consider Eq. (12) in the case of a circularly bent axial channel. This is the most important case, because a bent channel of arbitrary shape can be approximated by pieces of circles. The cylindrical coordinate system which we use is plotted in Fig. 2. The fast longitudinal motion of the particle in the channel corresponds to the rotation of the particle relative to the axis $z$. The slow transverse motion takes place in the plane $(\rho ; z)$. The potential of the channel depends only on the coordinates $(\rho, z)$ and does not depend on the angle $\varphi$. Therefore let us consider the solution of the Dirac equation (12) in the form

$$
\Psi=\exp \{i M \varphi\} \Psi(\rho ; z),
$$

where $M$ is the angular momentum of the particle in $z$ direction.

Substituting (15) into (12), we derive the following form of the Dirac equation for the motion in the plane $(\rho ; z)$ :

$$
\begin{aligned}
&\left\{-\frac{\hbar^{2}}{2 m}\left[\frac{1}{\rho} \frac{\partial}{\partial \rho}\left(\rho \frac{\partial}{\partial \rho}\right)+\frac{\partial^{2}}{\partial z^{2}}\right]\right.\left.+\frac{M^{2}}{2 m \rho^{2}}+U(\rho ; z)\right\} \\
& \times \Psi(\rho ; z)=\frac{p^{2}}{2 m} \Psi(\rho ; z) .
\end{aligned}
$$

This equation can be simplified if one notes that the potential $U(\rho ; z)$ depends in fact only on the local radial transverse coordinate of the particle $r_{\perp}^{\prime}$. We will suppose that the potential $U(\rho ; z)$ has local axial symmetry and

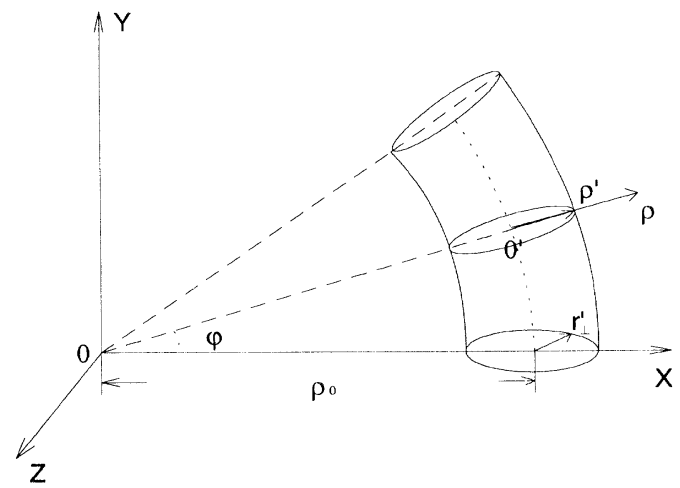

FIG. 2. The coordinate system for the bent axial channel. 
therefore $U(\rho ; z)=U\left(r_{\perp}^{\prime}\right)$ (see Fig. 2). Then, substituting

$$
\begin{aligned}
& M=p_{\|}\left(\rho_{0}+\rho_{0}^{\prime}\right), \\
& \rho=\rho_{0}+\rho^{\prime},
\end{aligned}
$$

where $\rho_{0}^{\prime}$ is the initial local coordinate of the particle in the channel, and taking into account that $\rho_{0} \gg \rho^{\prime} ; \rho_{0}^{\prime}$, we derive

$$
\begin{aligned}
-\frac{\hbar^{2}}{2 m}\left\{\frac{\partial^{2}}{\partial \rho^{\prime 2}}+\right. & \left.\frac{\partial^{2}}{\partial z^{2}}\right\} \Psi\left(r_{\perp}^{\prime} ; \rho^{\prime}\right) \\
& +\left\{U\left(r_{\perp}^{\prime}\right)-\frac{\varepsilon_{\|}}{\rho_{0}} \rho^{\prime}\right\} \Psi\left(r_{\perp}^{\prime} ; \rho^{\prime}\right) \\
& =\left(\varepsilon_{\perp}-\varepsilon_{\|} \frac{\rho_{0}^{\prime}}{\rho_{0}}\right) \Psi\left(r_{\perp}^{\prime} ; \rho^{\prime}\right) .
\end{aligned}
$$

Equation (18) differs from (14), which was derived for linear channeling. It contains additional centrifugal potential terms in both sides of the equation. Comparing these terms in (18) with the typical depth $U_{0}$ of the potential $U\left(r_{\perp}\right)$ we come to the conclusion that if the condition (4) is fulfilled then the centrifugal potential terms are small and can be neglected. In this case Eq. (18) coincides with the one obtained for the linear channeling case. It proves that channeling in a bent crystal has to take place indeed if condition (4) is fulfilled.

Equation (18) determines the specific features of the channeling process in a bent crystal, which we discuss in the next section.

\section{SPECIFIC FEATURES OF CHANNELING IN A BENT CRYSTAL}

\section{A. The effective potential of the transverse motion}

The motion of the particle in the transverse plane is determined by the effective potential acting on the particle. This potential appears in the equation of motion (18). Indeed the first term on the left hand side of (18),

$$
-\frac{\hbar^{2}}{2 m}\left\{\frac{\partial^{2}}{\partial \rho^{\prime 2}}+\frac{\partial^{2}}{\partial z^{2}}\right\}=-\frac{\hbar^{2}}{2 m} \Delta_{\vec{r}_{\perp}^{\prime}}
$$

is the transverse kinetic energy of the particle into the channel. While the second term is the effective potential energy

$$
U_{e f}\left(r_{\perp}^{\prime} ; \rho^{\prime}\right)=U\left(r_{\perp}^{\prime}\right)-\frac{\varepsilon_{\|}}{\rho_{0}} \rho^{\prime}=U\left(r_{\perp}^{\prime}\right)-\frac{\varepsilon_{\|}}{\rho} r_{\perp}^{\prime} \cos \varphi^{\prime} .
$$

Here we have used the local azimuthal coordinates $r_{\perp}^{\prime} ; \varphi^{\prime}$ of the particle in the channel. They are connected with $\rho^{\prime}$ and $z$ as $\rho^{\prime}=r_{\perp}^{\prime} \cos \varphi^{\prime} ; z=r_{\perp}^{\prime} \sin \varphi^{\prime}$. The cases of $\cos \varphi^{\prime}=0$ and $\cos \varphi^{\prime}=\pi$ correspond to the parallel and antiparallel orientation of the vectors $\overrightarrow{r^{\prime}}$ and $\overrightarrow{\rho^{\prime}}$. The effective energy contains two parts - the transverse potential of the channel $U\left(r_{\perp}^{\prime}\right)$ and the centrifugal potential contribution. The latter term increases with the energy of the particle in the channel and is inversely propor- tional to the channel's curvature. In the limit of the linear channel $\left(\rho_{0} \longrightarrow \infty\right)$ this contribution vanishes. The transverse energy of the particle in the channel depends on the initial coordinate of the particle in the channel $\rho_{0}^{\prime}$, which is described by the corresponding term in the right hand side of (18).

Let us now discuss in more detail the behavior of the effective potential (19). The potential $U\left(r_{\perp}^{\prime}\right)$ in (19) has two parts,

$$
U\left(r_{\perp}^{\prime}\right)=V\left(r_{\perp}^{\prime}\right)+\frac{M_{\perp}^{2}}{2 m r_{\perp}^{\prime 2}},
$$

where $V\left(r_{\perp}^{\prime}\right)$ is the transverse potential of the axial channel and $M_{\perp}$ is the angular momentum of the particle's rotation in the channel. Vibrations of atoms forming the channel influence the shape of the transverse potential $V\left(r_{\perp}^{\prime}\right)$. It makes the shape of $V\left(r_{\perp}^{\prime}\right)$ slightly different at different temperatures. The shape of $V\left(r_{\perp}^{\prime}\right)$ is well known in many crystals. There are analytic approximations for the transverse potential $V\left(r_{\perp}^{\prime}\right)$ (see, e.g., [17]), which depend both on crystal parameters and temperature. The shape of the effective potential (19) depends on many variables: $M_{\perp}, m, \varepsilon_{\|}, \rho_{0}$, and the angular position $\varphi$ of the particle in the channel $\left(\rho^{\prime}=r_{\perp} \cos \varphi\right)$. Figures $3(\mathrm{a})$ and $3(\mathrm{~b})$ illustrate the potential curves $U_{\text {ef }}\left(r_{\perp}^{\prime} ; \rho^{\prime}\right)$ for the cases $M_{\perp}=0$ and $M_{\perp}=100$ a.u. (the solid lines). The three-dimensional plot (the lower part) as well as the central cut (the upper part) of the effective potentials are given in these figures. The effective potential $V\left(r_{\perp}^{\prime}\right)$ shown in the upper parts of Figs. 3(a) and 3 (b) by the dashed line is calculated for $300 \mathrm{GeV}$ electrons aligned with the $\langle 110\rangle$ axis of the Ge crystal, and is at a temperature of $280 \mathrm{~K}$. The curvature of the channel is equal to $1 \mathrm{~cm}$. The asymptote of $U_{e f}\left(r_{\perp}^{\prime} ; \rho^{\prime}\right)$, which is determined by the second term in (19), is shown by the dotted line in the upper parts of Figs 3(a) and 3(b). The main difference between the two cases is the spiky feature in Fig. 3(b). This feature originates from the second term in (20), being the centrifugal energy of the particle rotating in the axial channel. Physically this means that the particle rotating in the axial channel is not classically allowed to penetrate to the center of the potential.

The case of planar channeling is very similar to the one considered above. In the planar channel the particle has two dimensions for fast quasi-free motion parallel to the crystal plane. The motion of the particle in the direction perpendicular to the crystal plane is governed by the crystal potential. In the bent channel one of the directions of the quasi-free fast motion becomes bent too. Choosing the $z$ direction as that for which the particles are bent and performing some transformations, which are very similar to those we did deriving (18), one obtains

$$
\begin{gathered}
\left\{-\frac{\hbar^{2}}{2 m} \frac{d^{2}}{d x^{\prime 2}}+U\left(x^{\prime}\right)-\frac{\varepsilon_{z}}{\rho_{0}} x^{\prime}\right\} \Psi\left(x^{\prime}\right) \\
=\left(\varepsilon_{\perp}-\varepsilon_{z} \frac{x_{0}^{\prime}}{\rho_{0}}\right) \Psi\left(x^{\prime}\right) \\
\varepsilon=\varepsilon_{y}+\varepsilon_{z}+\varepsilon_{\perp}, \quad m=m_{0} \gamma, \quad \varepsilon_{z}=\frac{m v_{z}^{2}}{2}
\end{gathered}
$$


Here $x^{\prime}$ is the local coordinate of the particle in the channel, $\varepsilon_{z}$ is the kinetic energy of the particle in $z$ direction, $\rho_{0}$ is the curvature radius, and $x_{0}^{\prime}$ is the initial coordinate of the particle in the channel.

The condition (4) in the case of planar channeling must be replaced by

$$
\frac{\varepsilon_{z} R}{U_{0} \rho_{0}} \ll 1
$$

Under this condition the centrifugal potential terms in (21) become negligible. The condition (22) can be much better fulfilled than (4), because $\varepsilon_{z}$ can be much smaller than the total energy $\varepsilon \approx \varepsilon_{y}$. The effective transverse potential for Eq. (21) is plotted in Fig. 4. This figure shows the transverse potential at room temperature in a bent Ge crystal for the three neighboring $\langle 110\rangle$ planes in comparison with that in crystal without bending. The energy of the electron is equal to $50 \mathrm{GeV}$ and the curvature of the channel is $1 \mathrm{~cm}$.
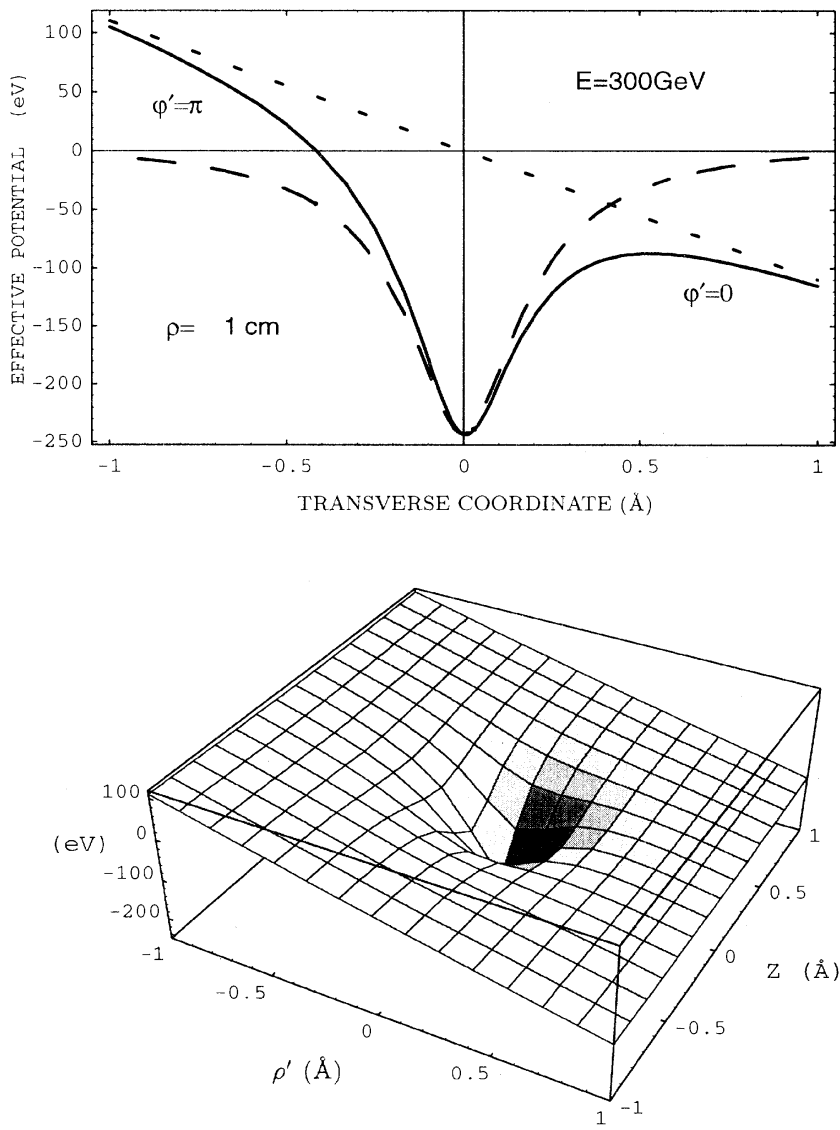

\section{B. The polarization of the transverse energy states}

Equations (18) and (21) are analogous to the equations of a two- or one-dimensional atom placed in the external uniform electric field. This analogy lets us make some conclusions about the transverse motion of the particle. Moving along the channel, the particle populates one of the discrete levels in the transverse potential. In a bent crystal these energy levels become shifted and split, compared to the linear channel case, like the energy levels of an atom in an external electric field.

This splitting can be calculated as [27]

$$
\begin{aligned}
& \Delta \varepsilon_{n ; M_{\perp}}=-\frac{1}{2} \alpha_{n ; M_{\perp}}\left(\frac{\varepsilon_{\|}}{\rho}\right)^{2}, \\
& \alpha_{n ; M_{\perp}}=-\frac{1}{2} \sum_{n^{\prime}}\left\{\frac{\left|\left\langle n^{\prime} ; M_{\perp}+1\left|r_{\perp}^{\prime}\right| n ; M_{\perp}\right\rangle\right|^{2}}{\varepsilon_{n ; M_{\perp}}-\varepsilon_{n^{\prime} ; M_{\perp}+1}}\right. \\
&\left.+\frac{\left|\left\langle n^{\prime} ; M_{\perp}-1\left|r_{\perp}^{\prime}\right| n ; M_{\perp}\right\rangle\right|^{2}}{\varepsilon_{n ; M_{\perp}}-\varepsilon_{n^{\prime} ; M_{\perp}-1}}\right\} .
\end{aligned}
$$
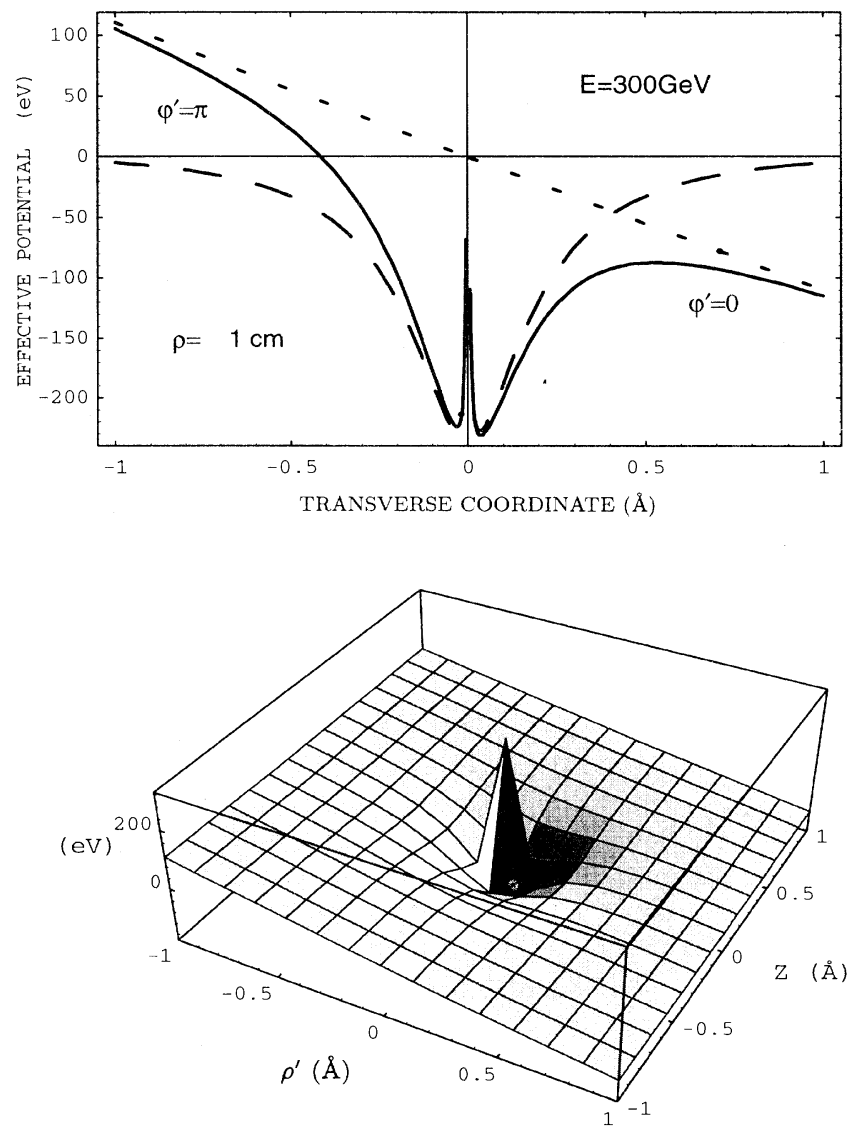

FIG. 3. (a) The effective transverse potential of the axial channel in Ge: axis $\langle 110\rangle ; M_{\perp}=0 ; T=280 \mathrm{~K} ; \varepsilon_{\|}=300 \mathrm{GeV}$. The upper part of the figure shows the central cut of the effective potential. The lower part demonstrates the dimensional plot of $U_{e f}\left(r_{\perp}^{\prime} ; \rho^{\prime}\right)$. The units used in the lower part of the figure are the same as in the upper one. (b) The effective transverse potential of the axial channel in Ge: axis $\langle 110\rangle ; M_{\perp}=100 ; T=280 \mathrm{~K} ; \varepsilon_{\|}=300 \mathrm{GeV}$. The upper part of the figure shows the central cut of the effective potential. The lower part demonstrates the dimensional plot of $U_{e f}\left(r_{\perp}^{\prime} ; \rho^{\prime}\right)$. The units used in the lower part of the figure are the same as in the upper one. 


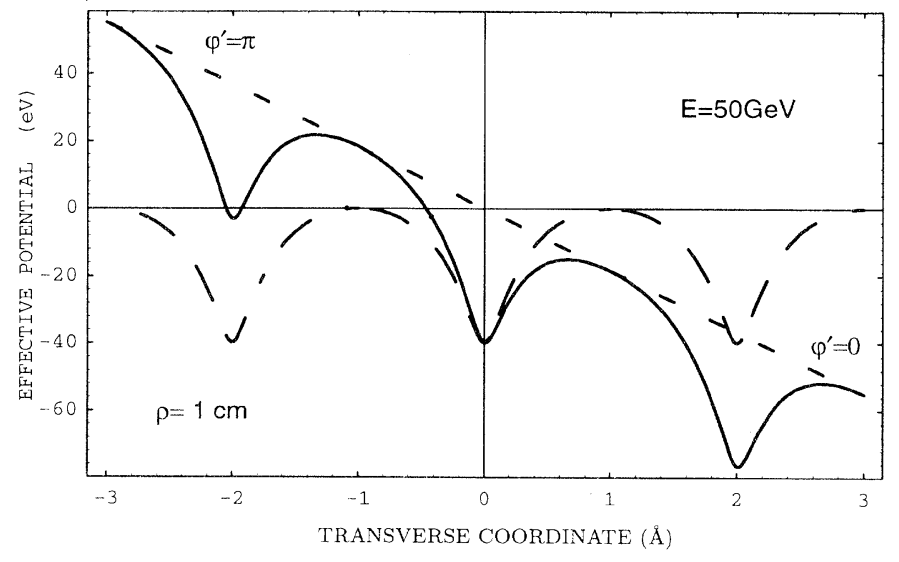

FIG. 4. The effective transverse potential of three successive planar channels in $\mathrm{Ge}$ : plane $\langle 110\rangle$; room temperature; $\varepsilon=50 \mathrm{GeV}$.

In (24) $\alpha_{n ; M_{\perp}}$ is the polarizability of the state $\left|n ; M_{\perp}\right\rangle$. The summation in (24) is performed over all discrete and continuous states of the transverse motion. The transitions $M_{\perp} \longrightarrow M_{\perp}+1 ; M_{\perp}-1$ only contribute to the polarizability $\alpha_{n ; M_{\perp}}$.

The states of the transverse motion become polarized due to the bending of the channel. The induced dipole moment of the state with the principal quantum number $n$ and the angular momentum $M_{\perp}$ is equal to

$$
D_{n ; M_{\perp}}=e \alpha_{n ; M_{\perp}} \frac{\varepsilon_{\|}}{\rho} .
$$

Estimating the polarizability $\alpha_{n ; M_{\perp}}$ as

$$
\alpha_{n ; M_{\perp}} \sim \frac{R^{2}}{U_{0}}
$$

one finds

$$
D_{n ; M_{\perp}} \sim e R\left(\frac{\varepsilon_{\|} R}{U_{0} \rho}\right)=e R C \ll e R .
$$

This condition shows that if (4) is fulfilled then the polarization of the transverse states is small. The induced dipole moment $D_{n, M_{\perp}}$ is much smaller than typical values of the transition dipole matrix elements between states of the transverse motion.

\section{The tunneling effect}

The right hand wing of the effective transverse potential of a given bent channel has a barrier (see Figs. 3 and 4). Therefore a particle captured by the potential can leave the channel by tunneling under the potential barrier in the effective potential.

Let us estimate the probability of this process. This can be done using different methods. It is obvious that the tunneling of the particle with the large relativistic mass can be considered as quasiclassical. Therefore the probability of dechanneling from a certain level in the transverse potential is equal to the probability of the particle's tunneling under the barrier, which can be calculated quasiclassically [27]. It is important to know the shape of the potential in this calculation. The calculation can be done analytically if one takes a simple transverse potential such as the oscillatory, square-well, or cutoff Coulomb potential with the appropriate parameters. The result of the calculation for the square-well potential is given by the expression

$$
W \sim \exp \left\{-\frac{4}{3} \frac{\varepsilon_{\perp}}{\varepsilon_{\|}} \frac{\rho \sqrt{2 m\left|\varepsilon_{\perp}\right|}}{\hbar}\right\} .
$$

In fact this result is slightly sensitive to the chosen shape of the transverse potential of the channel. The shape of the potential determines the preexponential factor omitted in (27), while the exponential factor turns out to be the same. One can also derive (27), approximating the potential barrier by the zero-range potential.

The quantity $\varepsilon_{\perp}$ in (27) is the binding energy of the particle in the transverse potential, which can be estimated as $\varepsilon_{\perp} \sim U_{0}$. The momentum appearing in the exponent is approximately equal to $\sqrt{2 m\left|\varepsilon_{\perp}\right|} / \hbar \sim 1 / R$. Then one obtains that the expression in the exponent is proportional to $1 / C$, which is large if the condition (4) is fulfilled. This result shows that the probability of dechanneling due to the tunneling effect turns out to be exponentially small. It becomes large only when the channeling effect itself is practically absent.

The model potentials which were used for the estimation of the probability of the tunneling effect are also very helpful in obtaining other characteristics of the channeling process in a bent crystal, e.g., the condition channeling and the polarization of the transverse energy states.

\section{The probability of the channeling process}

Let us now estimate the probability of the particle to be captured by the field of a bent channel. We only consider the case where the particle moves initially parallel to the direction of the channel. If the channel is linear then the particle populates one of the discrete levels in the effective transverse potential. In the case of a bent channel the continuous spectrum of the energy levels coexists with the spectrum of the quasidiscrete levels (see the potential in Fig. 4). The channeling effect occurs only if the particle populates one of the levels of the quasidiscrete spectrum. Assuming that all the states in the discrete and the continuous spectra are being initially populated with equal probability, we come to the conclusion that the probability for channeling can be estimated as

$$
W=\frac{N_{D}}{N_{D}+N_{C}}
$$

where $N_{D}$ and $N_{C}$ are the number of states in the discrete and continuous spectra, respectively.

Let us investigate the dependency of $W$ on the param- 
eters of the system. The number of states in the continuous spectrum can be estimated from the density of these states:

$$
\begin{array}{r}
N_{C}=2 \times \int_{0}^{p_{\max }} \frac{d p 2 R}{2 \pi}, \\
p_{\max }=\sqrt{4 m\left|U_{\max }\right|} .
\end{array}
$$

The factor 2 in front of the integral takes into account the spin of the electron; $p_{\max }$ is the maximum momentum the electron can have in the effective potential of a given channel; $U_{\max }$ is the maximum value in the right hand wing of the effective potential of a given channel (see Fig. 4).

The number of discrete levels in the potential well formed by the effective transverse potential can be easily estimated, if one approximates this well by a square-well potential with the appropriate parameters. The number of states in a square-well potential of depth $U$ and width $a$ is [4]

$$
N_{D}=\sqrt{\frac{2 m a^{2} U}{\pi^{2}}} .
$$

In our case the depth of the potential is $U \sim U_{0}-$ $U_{\max } \approx U_{0}$. The width of the potential $a$ is determined by the radial position of $U_{\max }$. Approximating the tail of the potential of the channel by $Z_{e f} / x^{\prime}$ we find that the coordinate of $U_{\max }$, and therefore $a$, is equal to $\left(Z_{e f} \rho / \varepsilon_{\|}\right)^{1 / 2}$. Using these estimates in (30) and then substituting $N_{D}$ and $N_{C}$ in (28), we derive the following expression for $W$ :

$$
W=\frac{1}{1+a C^{3 / 4}} .
$$

Here the parameter $C$ is the main parameter of the problem defined in (4). The constant $a=\left(4 U_{0} R / Z_{\text {ef }}\right)^{1 / 4}$ depends on the potential characteristics and can be considered as an empirical constant. Substituting the typical parameters $U_{0} \sim 4, R \sim 2$, and $Z_{\text {ef }} \sim 2$ written in atomic units one derives that $a \sim 2$. The result (31) shows again that the probability of the channeling in a bent crystal is determined by $C$.

\section{E. Radiation by a particle channeled in a bent channel}

The radiation emitted by a particle moving in a bent channel differs from that in the case of a linear channel. The additional contributions to the radiation spectrum arise due to the curvature of the channel and disappear in the case of a linear channel.

Let us qualitatively discuss the origin of these contributions to the radiation spectrum. The first one is a result of the emission by the charged particle accelerated due to the bending of the particle's trajectory in the field of the crystal. This radiation is very similar to the traditional synchrotron radiation of the particle moving in the uniform magnetic field. Another contribution to the spectrum arises because of the rotation of the induced dipole moment of transverse states.

We have demonstrated that the polarization of the states of transverse motion cannot be large under channeling conditions. Therefore the contribution to the total radiation spectrum arising from the polarization of the transverse particle states turns out to be much smaller than the contribution from transitions between different levels of transverse motion. The latter mechanism exists also for linear channeling and has been investigated in detail [17-20].

In this paper we consider only the radiation generated by the additional acceleration in the field of a bent channel. This problem can be easily solved using the results of the theory of synchrotron radiation in a uniform magnetic field [26]. Replacing the magnetic field by the transverse force $\varepsilon_{\|} / \rho$ acting on the particle in a bent channel, we derive the spectrum

$$
\begin{gathered}
\frac{d N_{\omega}}{d \omega}=-\frac{e^{2} \gamma^{-2} L}{c^{2}}\left\{\int_{\xi}^{\infty} d \xi^{\prime} \Phi\left(\xi^{\prime}\right)\right. \\
\left.+\left(\frac{2}{\xi}+\frac{\omega}{\varepsilon} \chi \xi^{1 / 2}\right) \Phi^{\prime}(\xi)\right\} \\
\chi=\frac{\varepsilon_{\|}}{\rho} \frac{\gamma}{m_{0}^{2} c^{3}}, \quad \xi=\left(\frac{\omega}{\chi\left(\varepsilon_{\|}-\omega\right)}\right)^{2 / 3}, \quad \varepsilon_{\|}=m_{0} c^{2} \gamma .
\end{gathered}
$$

The value of the transverse force $\varepsilon_{\|} / \rho$ acting on the particle was obtained from the Dirac equation analysis performed in Sec. IIB. The spectrum (32) is only determined by the curvature of the crystal and the energy of the electron. Therefore the same spectra are emitted in different kinds of crystals with the same curvatures, if the projectile electron energies coincide.

We have used (32) to calculate the spectrum of emitted photons at different values of the relativistic parameter $\gamma$ and curvature $\rho$. The results are plotted in Fig. 5. The intensity in Fig. 5 is defined as $\omega d N / d \omega / L$ and given in $\mathrm{cm}^{-1}$. The photon frequency is plotted as $\omega / \varepsilon_{\|}$. This figure shows that with increasing electron energy the radiation intensity increases and the frequency maximum of the emitted photons is shifted to the higher values. With increasing channel curvature the intensity of the radiation decreases. These dependencies are qualitatively clear. The electron radiates more intensively when its acceleration is larger.

The derived intensities of radiation are rather large compared to those obtained for linear channeling [17]. For example, the maximum intensity of the radiation of the $150 \mathrm{GeV}$ electron aligned with $\langle 110\rangle$ in $\mathrm{Ge}$ at $T=100$ $\mathrm{K}$ is approximately equal to $20 \mathrm{~cm}^{-1}$. This intensity is achieved in a bent crystal for $50 \mathrm{GeV}$, when $\rho=10$, as is clear from Fig. 5. In this case the main parameter $C \sim 1$. In this case the main condition (4) becomes violated and the probability of channeling decreases rapidly, but it is still rather large, being on the level of $30 \%$, as follows from (31) and the estimates performed in the preceding section. At smaller curvatures (see curves $\rho=5$ $\mathrm{cm}$ and $\rho=2 \mathrm{~cm}$ in the upper part of Fig. 5) or higher electron energies (see curve $E=250 \mathrm{GeV}$ in the lower part of Fig. 5) the intensity of radiation in a bent crys- 

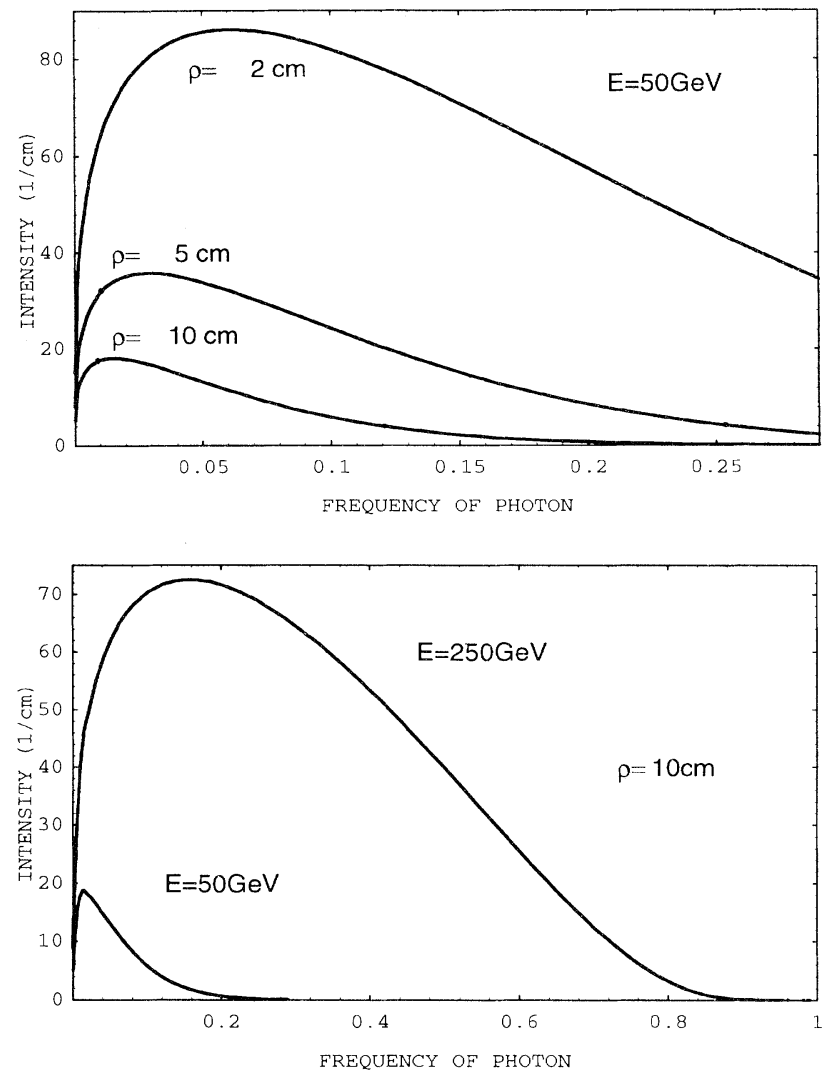

FIG. 5. The radiative spectra of an electron channeling in a bent crystal calculated at different energies and curvatures of the channel. The intensity is given in $\mathrm{cm}^{-1}$. The photon frequency is plotted as $\omega / \varepsilon_{\|}$.

tal substantially exceeds the intensity of radiation in the linear case. At the same time the probability of channeling decreases according to (31). As a result of these two opposite tendencies the effective intensity of the photon emission becomes comparable again with the intensity of the radiation of the electron in the linear channel, even if $C \sim 5$. The case $C \sim 5$ corresponds to the curve $\rho=2 \mathrm{~cm}$ in the upper part of Fig. 5 and to the one with $E=250 \mathrm{GeV}$ in the lower part. In this case the probability $W,(31)$, is approximately equal to $15 \%$.

The total radiative energy loss is determined by the parameter $\delta=\hbar \omega_{0} / \varepsilon_{\|}$, where $\omega_{0}=c \gamma^{4} / \rho$ is the frequency corresponding to the maximum in the photon distribution [26]. This parameter can be expressed in the form

$$
\delta=\frac{\hbar \omega_{0}}{\varepsilon_{\|}}=\frac{\gamma^{2}}{m_{0} c \rho}=C \frac{U_{o}}{m_{0} c^{2}} \frac{\hbar}{m_{o} c R} \gamma .
$$

[1] M.T. Robinson, Appl. Phys. Lett. 1, 49 (1962).

[2] M.T. Robinson and O.S. Oen, Appl. Phys. Lett. 2, 30 (1963); Phys. Rev. 132, 2385 (1963).

[3] J. Stark, Phys. Z. 13, 973 (1912).

[4] J. Lindhard, K. Dan. Vidensk. Selsk. Mat.-Fys. Medd. 34, No. 14 (1965).
If $\delta \sim 1$, then almost all energy of the particle is transferred to the emitted photons. The representation of $\delta$ in the form (33) shows that under the channeling conditions $\delta$ becomes comparable to unity at $\gamma \sim 10^{7}$, because $C \ll 1 ; U_{0} / m_{0} c^{2} \sim 10^{-4}$ and $\hbar / m_{0} c R \sim 10^{-2}$.

\section{SUMMARY}

We have considered the process of channeling in a bent crystal. Using simple qualitative arguments we have derived the criterion for channeling and proved it using the Dirac equation. It was shown that the centrifugal force acting on the particle in the bent crystal significantly alters the effective transverse potential for both axial and planar channeling. We estimated the probability of dechanneling of the particle due to its tunneling in the effective transverse potential and demonstrated that it is exponentially small if the condition for channeling is fulfilled. The total probability to observe channeling in a bent crystal is also estimated. It depends on the specific scaling parameter characterizing the process. Using the quasiclassical theory of synchrotron radiation we have calculated the spectra of photons being emitted by the channeled particle at different energies. We have considered the contribution to the total radiation spectrum due to the curvature of the channel and demonstrated that it becomes sizable for the energy range which is planned for the electron-positron colliders of the new generation.

Let us briefly mention some possible practical applications. Bent crystals can be used for different manipulations of the charged particle beams. One can think about "channeling lenses" for focusing beams or "channeling magnets" for bending of the charged particles. We have demonstrated that the channeling magnets can be used for generating synchrotronlike radiation.

Let us mention some questions left for further investigations. One can generalize the results to the case of a channel of arbitrary shape. It is also interesting to take into account the effects associated with the spin motion of the particle in the channel. A quite important problem is the description of the energy loss of electrons or positrons in a bent crystal. Analogously to the linear channeling case, rate equations which include the new radiation terms must be used for solving this problem.

\section{ACKNOWLEDGMENTS}

The authors are thankful to the Deutsche Forschungsgemeinschaft, the GSI, and BMFT for support.
[5] C. Lehmann and G. Leibfreid, J. Appl. Phys. 34, 2821 (1963).

[6] D.S. Gemmell, Rev. Mod. Phys. 46, 129 (1974).

[7] A. Schäfer, S. Graf, J. Augustin, W. Greiner and E. Uggerhoj, J. Phys. G 16, L131 (1990).

[8] J. Klenner, J. Augustun, A. Schäfer, and W. Greiner, 
Phys. Rev. A 50, 1019 (1994).

[9] O.R. Frisch and D.N. Olson, Phys. Rev. Lett. 3, 141 (1959).

[10] J. Kimball and N. Cue, Phys. Rep. 125, 69 (1985), and references therein.

[11] A.O. Aganyants, Yu.A. Vartanov, G.A. Vatapetyan, M.A. Kumakhov, Chr. Trikalinos, and Ya.V. Yaralov, Pis'ma Zh. Eksp. Teor. Fiz. 29, 554 (1979) [JETP Lett. 29, 505 (1979)].

[12] R.L. Swent, R.H. Pantell, M.J. Alguard, B.L. Berman, S.D. Bloom, and S. Datz, Phys. Rev. Lett. 43, 1723 (1979).

[13] M.A. Kumakhov, Phys. Lett. A 57, 17 (1976).

[14] V.G. Baryshevskii, Nuclear Optics of the Polarized Media (Minsk, 1976) (in Russian).

[15] J.U. Andersen, E. Bonderup, and R.H. Pantell, Annu. Rev. Nucl. Part Sci. 33, 453 (1983).

[16] B.L. Berman and S. Datz, in Coherent Radiation Sources (Springer-Verlag, Berlin, 1985), p. 165.

[17] V.N. Baier, V.M. Katkov, and V.M. Strakhovenko, Phys. Lett. A 114, 511 (1986); 117, 251 (1986); Zh. Eksp. Teor. Fiz. 92, 588 (1987) [Sov. Phys. JETP 65, 686 (1987)]; High Energy Electromagnetic Processes in Ori- ented Monocrystals (Nauka, Novosibirsk, 1989) (in Russian).

[18] V.V. Tikhomirov, Phys. Lett. A 125, 411 (1987).

[19] Yu.V. Kononetz and V.A. Ryabov, Nucl. Instrum. Methods Phys. Res. Sect. B 48, 269 (1990).

[20] J. Augustin, Ph.D. thesis, University of Frankfurt am Main, 1993.

[21] S. P. Moller, E. Uggerhoj, H.W. Atherton, M. Clément, N. Doble, K. Elsener, L. Gatignon, P. Grafstrom, M. Hage-Ali, and P. Siffert, Phys. Lett. B 256, 91 (1991).

[22] A.F. Elishev et al., Phys. Lett. 88B, 387 (1979).

[23] J.F. Bak, G. Melchart, E. Uggerhoj, I.S. Forster, P.R. Jensen, H. Mads Boll, S.P. Moller, H. Nielsen, G. Petersen, H. Schiott, J.J. Gross, and P. Siffert, Phys. Lett. 93B, 505 (1980).

[24] Relativistic Channeling, edited by A. Carrigan and J. Ellison (Plenum, New York, 1987).

[25] A. Schäfer and W. Greiner, J. Phys. G 17, L217 (1991).

[26] L.D. Landau and E.M. Lifshitz, Quantum Electrodynamics (Pergamon Press, New York, 1965).

[27] L.D. Landau and E.M. Lifshitz, Quantum Mechanics (Pergamon Press, New York, 1965). 

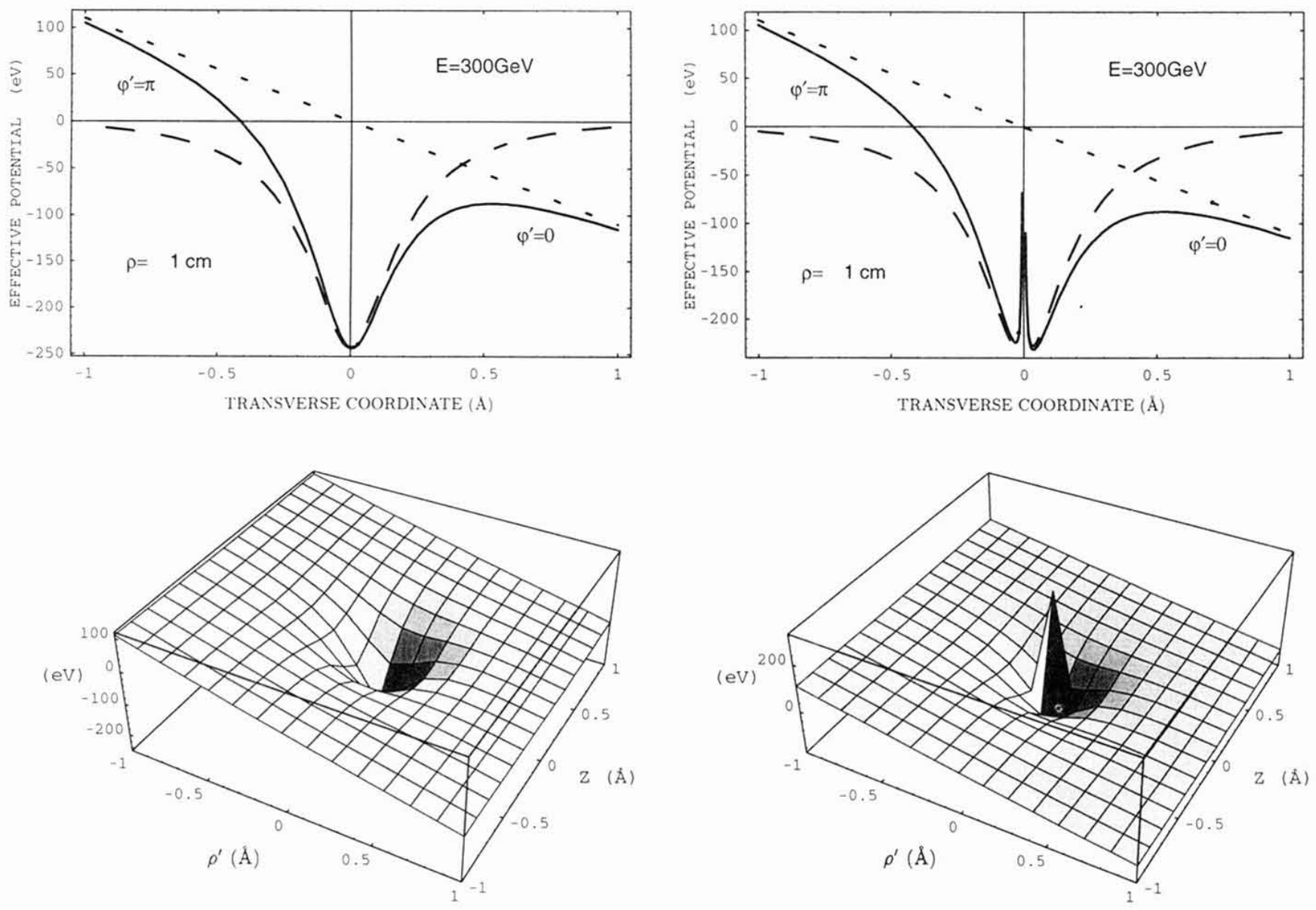

FIG. 3. (a) The effective transverse potential of the axial channel in Ge: axis $\langle 110\rangle ; M_{\perp}=0 ; T=280 \mathrm{~K} ; \varepsilon_{\|}=300 \mathrm{GeV}$. The upper part of the figure shows the central cut of the effective potential. The lower part demonstrates the dimensional plot of $U_{e f}\left(r_{\perp}^{\prime} ; \rho^{\prime}\right)$. The units used in the lower part of the figure are the same as in the upper one. (b) The effective transverse potential of the axial channel in Ge: axis $\langle 110\rangle ; M_{\perp}=100 ; T=280 \mathrm{~K} ; \varepsilon_{\|}=300 \mathrm{GeV}$. The upper part of the figure shows the central cut of the effective potential. The lower part demonstrates the dimensional plot of $U_{e f}\left(r_{\perp}^{\prime} ; \rho^{\prime}\right)$. The units used in the lower part of the figure are the same as in the upper one. 